\title{
Eigen Combination of Colour and Texture Informations for Image Segmentation
}

\author{
D. Attia, C. Meurie, and Y. Ruichek \\ Université de Technologie de Belfort-Montbéliard, \\ Institut Régional Supérieur du Travail Educatif et Social de Bourgogne, \\ Laboratoire Systémes et Transports, \\ 13 rue Ernest-Thierry Mieg, 90010 Belfort Cedex, France \\ \{dhouha.attia, cyril.meurie, yassine.ruichek\}@utbm.fr
}

\begin{abstract}
In this paper, we present a new combination of colour and texture informations for image segmentation. This technique is based on principal components analysis of a $3 \mathrm{D}$ points cloud, followed by an eigenvalues analysis. A set of colour gradients (morphological, Di-Zenzo) and texture gradients (Gabor, three Haralick attributes, Alternative Sequential Filter (ASF)) are used to test the proposed combination. The segmentation is performed using a hybrid gradient based watershed algorithm. The major contribution of this work consists in combining locally colour and texture informations using an adaptive and non parametric approach. The proposed method is tested on 100 images from the Berkley dataset [1] and evaluated with the Mean Square Error (MSE), the Variation of Information (VI) and the Probabilistic Rand Index (PRI).
\end{abstract}

\section{Introduction}

One of the most challenging problems in computer vision concerns image segmentation. If we consider colour and texture informations, the segmentation methods can be divided into three classes. The first one regroups those using only texture information [234], while the second class contains segmentation methods which use only colour information 56678 . In spite of satisfactory results of these approaches, the combination of colour and texture informations leads generally to obtain best results and increases the robustness of segmentation [91011]. In this paper, we propose a non parametric method to combine colour and texture attributes. This combination allows defining a structural gradient that will be used in watershed algorithm. This approach is based on principal components analysis (PCA) of a 3D points cloud formed by colour and texture descriptors, followed by an eigenvalues analysis. This paper is organized as follows : in section 2 , the segmentation step by watershed algorithm based on a structural gradient is presented. Then, colour and texture combination approach is detailed. In section 3, we describe extensive experiments carried out on 100 images from the BSDB dataset to test and validate the proposed method. 


\section{Image Segmentation Using Colour and Texture Informations Collectively}

In this section, a short introduction of watershed algorithm based on colour and texture gradients is presented. Then, an existing fixed combination based approach is briefly described. Finally the proposed approach based on PCA and eigenvalues analysis is detailed.

\subsection{Watershed Algorithm Based on Colour-Texture Gradients}

Watershed algorithm segments image into watershed regions [12 13. Considering the input image (gradient image) as a topographic surface, each seed of the input region (calculated with an optimal density) can be viewed as the point to which water falling on the surrounding region drains. The boundaries of watersheds lie on tops of ridges. In this paper, a new combination of colour and texture informations is proposed, in order to provide a hybrid gradient which will be the input of watershed algorithm. Before detailing the combination, different colour and texture gradients that will be used for tests are introduced.

Texture is an important perceptual information that is generally extracted using mathematical tools. In literature, four main classes of texture descriptors are discriminated: geometric attributes, descriptors based on spatial texture models, spatio-frequential and statistic attributes. In the present paper, we choose to use the following descriptors: Gabor transformation (spatio-frequential attribute) [14 15], three Haralick parameters (second order statistic attributes) [16 17] and an Alternate Sequential Filter (a transformation from mathematical morphology to calculate a texture gradient) [18. Considering colour information, we use Di-Zenzo gradient based on the first derivative of the initial image (see [19] for more details) and morphological gradient which corresponds to the subtraction between dilatation and erosion of the initial image (using a lexicographic order).

In literature, one can find an interesting approach combining colour and texture informations [20. The combination process is based on a set of operations derived from mathematical morphology. This technique uses collectively colour and texture informations to generate a structural gradient used in image segmentation. As expressed in equation 1] this gradient is obtained by a fixed combination of colour and texture gradients. In equation 1 $Q_{c o l}$ and $Q_{t e x}$ represent respectively colour and textural gradients. The textural gradient is obtained using following steps: filtering, definition of texture layer, and granulometric analysis. $\alpha$ represents the combination parameter, which is chosen between 0 and 1 . Even if this approach gives satisfactory results, its major drawback concerns the choice of the optimal value of the parameter $\alpha$ for each image. Furthermore, $\alpha$ is global for the entire image, and thus the combination does not take into account pertinent local information.

$$
Q_{\text {struc }}(I)=(1-\alpha) Q_{\text {tex }}(I)+\alpha Q_{\text {col }}(I)
$$


Recent work in combining colour and texture descriptors were presented in [21]. In this work, the authors were mostly concerned with detecting contours then image segmentation. Their contour detection is based on combining multiple local cues into a globalization framework based on spectral clustering. The contour detection results are injected into the segmentation step. The segmentation algorithm consists of a generic approach for transforming any contour detector into a hierarchical region tree. The authors use four features in their approach : after transforming the input image into CIE Lab colorspace, they extract brightness, colour $a$ and colour $b$ channels. The fourth feature channel is a texture channel, which assignes to each pixel a texton $i d$. Then, for each feature channel, an oriented gradient $G(x, y, \theta)$ is computed by placing at location $(x, y)$ a circular disc split into two half-discs by a diameter at angle $\theta$. The combination step of these local cues is given by equation 2 .

$$
m P b(x, y, \theta)=\sum_{s} \sum_{i} \alpha_{i, s} G_{i, s, \sigma(i, s)}(x, y, \theta)
$$

where $m P b$ is the multiscale predicted boundary detector, $s$ refers to scales, $i$ indexes feature channels (brightness, colour $a$, colour $b$, texture), and $G_{i, s, \sigma(i, s)}$ $(x, y, \theta)$ measures the histogram difference in channel $i$ between two halves of a disc of radius $\sigma(i, s)$ centred at $(x, y)$ and divided by a diameter at angle $\theta$. The parameters $\alpha_{i, s}$ weight the relative contribution of each gradient signal. Contrary to this linear combination of local cues, the optimal mixing function could be non linear. Thus, in 22], each cue was treated as an expert for a certain class of boundary and a set of classifiers (such as Classification Trees and SVM) were used to combine the various cues. Even if this approach gives satisfactory results, its major difficulty consists of the dependency of the segmentation step to the boundaries detection. A second limit concerns the choice of the optimal mixture function of the various local cues.

\subsection{PCA Based Colour-Texture Combination : Eigen Combination}

We propose a novel combination based on Principal Components Analysis (PCA) of a 3D points cloud, followed by an eigenvalues analysis. Principal Components Analysis is a technique which uses geometric and graphic representations to describe the dispersion of a dataset (observations). This dataset is assimilated to a points cloud $P$ composed by $m$ quantitative variables having $n$ unities (called also subjects):

$$
P^{s}=\left\{\left(G_{i 1}^{s}, \ldots G_{i j}^{s} \ldots G_{i m}^{s}\right), \forall i \in 1 \ldots n\right\}
$$

where index $i$ corresponds to subject $i$ and index $j$ corresponds to variable $j$ such as :

$$
p_{. j}=\left(p_{1 j} \cdots p_{n j}\right)
$$

By representing the subjects, we can determine which ones are similar. On the other hand if we represent the variables, we can study structures of linear links 
within the 3D points cloud, and then determine correlated variables [23 24]. Based on linear algebra, PCA technique aims also to extract axes which conserve the maximum of information [24]. The first step consists in calculating the covariance matrix of the points cloud. Then, eigenvalues and associated eigenvectors of the covariance matrix are extracted. Eigenvalues give the variation of the points cloud along principal components which are obtained by eigenvectors. Principal Components Analysis is generally used to eliminate the correlation of initial data and to reduce their size. In computer vision, PCA is used for image classification [25, image compression [26 27] and objects recognition [28]. In the present paper, PCA technique permits to determine axis which conserve the maximum of information (pertinent colour and texture informations). Thus, the proposed approach generates a novel structural hybrid gradient which will be used as an input of watershed algorithm.

Let $I$ be the initial image, $s$ a pixel in the image $I$ and $P^{s}$ is the associated $3 \mathrm{D}$ points cloud generated for the pixel $s . P^{s}$ contains the colour and texture values of $n$ gradients (in our case, $n$ represents the number of the used colour and texture descriptors). Each gradient $\left(G_{i .}^{s}\right)_{i=1, \ldots, n}$ is calculated in a colour space $E_{1} E_{2} E_{3} . m=3$, is the number of the variables, and $\forall i \in\{1, \ldots, n\} G_{i}^{s} \in \mathbb{R}^{3}$. Therefore, the local 3D points cloud can be defined as below :

$$
\begin{gathered}
P^{s}=\left\{\left(G_{i 1}^{s}, G_{i 2}^{s}, G_{i 3}^{s}\right), \forall i \in 1 \ldots n\right\} \\
P^{s}=\left(G_{1 .}^{s}, G_{2 .}^{s}, \ldots, G_{n .}^{s}\right)
\end{gathered}
$$

The covariance matrix of the $3 \mathrm{D}$ points cloud $P^{s}$ is generated using the following equation :

where :

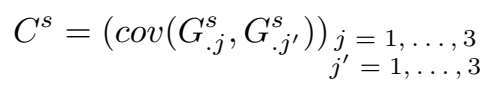

$$
\begin{gathered}
\operatorname{cov}\left(G_{. j}^{s}, G_{. j^{\prime}}^{s}\right)=\frac{1}{n} \sum_{i=1}^{n}\left(G_{i j}^{s}-\bar{G}_{. j}^{s}\right)\left(G_{i j^{\prime}}^{s}-\bar{G}_{. j^{\prime}}^{s}\right) \\
\bar{G}_{. j}^{s}=\frac{1}{n} \sum_{i=1}^{n} G_{i j}^{s}
\end{gathered}
$$

According to linear algebra rules, there are two local matrices $L^{s}$ and $D^{s}$ such as :

$$
\left(L^{s}\right)^{-1} C^{s} L^{s}=D^{s}
$$

Let $\lambda_{1}^{s}, \lambda_{2}^{s}$ and $\lambda_{3}^{s}$ be the eigenvalues of the local covariance matrix $C^{s}$ such as $\lambda_{3}^{s} \leq \lambda_{2}^{s} \leq \lambda_{1}^{s}$. Let $V_{1}^{s}, V_{2}^{s}, V_{3}^{s}$ be the associated eigenvectors. Therefore, the matrices $D^{s}$ and $L^{s}$ are expressed by the following equations :

$$
D^{s}=\left(\begin{array}{ccc}
\lambda_{1}^{s} & 0 & 0 \\
0 & \lambda_{2}^{s} & 0 \\
0 & 0 & \lambda_{3}^{s}
\end{array}\right)
$$




$$
L^{s}=\left(V_{1}^{s} V_{2}^{s} V_{3}^{s}\right)
$$

In order to determine the principal components that maximize local information, the eigenvalues are compared according to three cases listed as follows :

Case $1 \lambda_{1}^{s} \gg \lambda_{2}^{s}$ : One can conclude that it exists only one axis (direction of $V_{1}^{s}$ ) around which the $3 \mathrm{D}$ points cloud is concentrated. In this case, only the third principal component maximizing the local information is chosen. Thus, the new value $\hat{I}(s)$ of the pixel $s$ is given by the following mathematical formulation:

$$
\hat{I}(s)=\operatorname{argmax}_{i \in\{1, \ldots, n\}}\left\{G_{i 1}\right\}
$$

Case $2 \lambda_{3}^{s} \ll \lambda_{2}^{s}$ : In this case, $\lambda_{1}^{s}$ and $\lambda_{2}^{s}$ have the same order of the magnitude and the $3 \mathrm{D}$ points cloud is assimilated to a plane formed by the eigenvectors $V_{1}^{s}$ and $V_{2}^{s}$ (respectively associated to the eigenvalues $\lambda_{1}^{s}$ and $\lambda_{2}^{s}$ ). The eigenvector $V_{3}^{s}$ constitutes the normal of the plane $\left(\widehat{V_{1}^{s}, V_{2}^{s}}\right)$. Therefore, there are two principal components conserving the maximum of local information. In this case, the new value $\hat{I}(s)$ of the pixel $s$ is given by the following equation:

$$
\hat{I}(s)=\operatorname{argmax}_{i \in\{1, \ldots, n\}}\left\{\frac{1}{2}\left(G_{i 1}^{s}+G_{i 2}^{s}\right)\right\}
$$

Case $3 \lambda_{1}^{s} \simeq \lambda_{2}^{s} \simeq \lambda_{3}^{s}$ : In this case, the local 3D points cloud is dispersed according to all directions. Thus, no information is privileged. All principal components are fairly considered, and the new value $\hat{I}(s)$ of the pixel $s$ is given by the following mathematical formulation:

$$
\hat{I}(s)=\operatorname{argmax}_{i \in\{1, \ldots, n\}}\left\{\frac{1}{3}\left(G_{i 1}^{s}+G_{i 2}^{s}+G_{i 3}^{s}\right)\right\}
$$

\section{Experiments and Discussion}

In this section, an evaluation of the proposed colour and texture combination is presented. The results are compared to those obtained by the fixed combination [20], described before. Extensive experiments are carried out on 100 images from the BSDB dataset [1] using Mean Square Error (MSE) and two other evaluation metrics used in [21]: the variation of information (VI) [29] and the Probabilistic Rand Index (PRI) [30]. In order to conclude on the effectiveness and the robustness of the proposed approach, tests including two colour gradients (Di-Zenzo and morphological) and five texture gradients (ASF, Gabor filter, Second Angular Moment (SAM) attribute, Coherence attribute and Variance attribute) are realized. Table 1presents the evaluation by MSE criteria of segmentation results obtained with both the fixed and the proposed combinations on ten images of the database.

For a better visualization, we present only the obtained results with a texture gradient calculated with the Variance attribute. This example shows the difficulty to choose the best value of the parameter $\alpha$ when a fixed combination is 
Table 1. Segmentation results of the fixed and proposed approaches on 10 images of the Berkley database ( $\alpha^{*}$ corresponds to optimal value of $\alpha$ parameter)

\begin{tabular}{|c|c|c|c|c|c|c|c|}
\hline \multirow{2}{*}{$\begin{array}{l}\text { Initial } \\
\text { Image }\end{array}$} & \multicolumn{6}{|c|}{ Fixed combination } & \multirow{2}{*}{$\begin{array}{l}\text { Proposed } \\
\text { approach }\end{array}$} \\
\hline & $\alpha=0$ & $\alpha=0.2$ & $\alpha=0.5$ & $\alpha=0.7$ & $\alpha=1$ & $\alpha^{*}$ & \\
\hline & 189.7 & 198.6 & 199.4 & 204.4 & 190.5 & & 179.9 \\
\hline 4 & 242.9 & 220.8 & 223.2 & 220.9 & 264.5 & & 203.5 \\
\hline & 85.4 & 86.3 & 76.7 & 90.1 & 89.9 & & 82.5 \\
\hline & 103.8 & 95.9 & 82.9 & 81.6 & 92.5 & & 90.3 \\
\hline & 106 & 195.6 & 141.2 & 145.3 & 721.3 & & 126.5 \\
\hline & 94.7 & 93.4 & 110.5 & 101.4 & 106.6 & & 91.9 \\
\hline & 169.3 & 159.8 & 114.4 & 127.1 & 116.4 & & 140.2 \\
\hline & 244.1 & 138.3 & 131.6 & 117.7 & 135.1 & & 112.8 \\
\hline & 123.2 & 115.4 & 112.6 & 79.1 & 78.7 & & 84.7 \\
\hline$x^{x}$ & 75.2 & 68.1 & 65.8 & 66.6 & 73.4 & & 41.9 \\
\hline
\end{tabular}

applied. Indeed, the value of this parameter is not the same for all images and must be redefined for each image to obtain an acceptable segmentation. Considering different values of the parameter $\alpha$, one can notice that the proposed approach obtains the second position or the third one when it is not in the first position, when MSE of the two methods are compared. Moreover, when the fixed combination obtains the first position, the gap with the proposed method is very low. For more details, table 2 presents the evaluation with the mean of MSE, the mean of PRI and the mean of VI on all images of the database for the proposed approach according to the used colour and texture gradients. One can conclude that the segmentation results are similar even if the best one is obtained with the texture variance gradient and Di-Zenzo colour gradient if we consider MSE criteria. If we consider measures of PRI metric, best results are obtained with the texture coherence gradient and the morphological colour gradient. Finally, considering VI metric, best results are obtained by texture variance gradient and morphological colour gradient.

In figure 1, segmentation results are illustrated for the two combinations (the fixed and proposed approaches) using the best colour and texture gradients (DiZenzo colour gradient and texture variance gradient considering the MSE metric of evaluation). Even if the results of the two combinations are similar for the second and third images, one can notice that the proposed method provides better results for the other images. For example, the segmentation of different 
Table 2. Segmentation results of the proposed approach on 100 images of the Berkley database

\begin{tabular}{|c|c|c|c||c|c|c|}
\hline \multirow{2}{*}{ Texture gradient } & \multicolumn{6}{|c|}{ Colour gradient } \\
\cline { 2 - 7 } & \multicolumn{1}{|c|}{ Morphological gradient } & \multicolumn{2}{|c|}{ Di-Zenzo gradient } \\
\cline { 2 - 7 } & MSE & PRI & VI & MSE & PRI & VI \\
\hline Gabor & 388,4 & 0.69 & 1.33 & 340,2 & 0.69 & 1.64 \\
\hline ASF & 394,8 & 0.69 & 1.31 & 334,5 & 0.70 & 1.66 \\
\hline SAM & 394,8 & 0.69 & 1.65 & 344,8 & 0.69 & 1.84 \\
\hline Coherence & 390,1 & $\mathbf{0 . 7 1}$ & 1.29 & 334,5 & 0.70 & 1.59 \\
\hline Variance & 381 & 0.70 & $\mathbf{1 . 2 5}$ & $\mathbf{3 2 4 , 7}$ & 0.70 & 1.57 \\
\hline
\end{tabular}
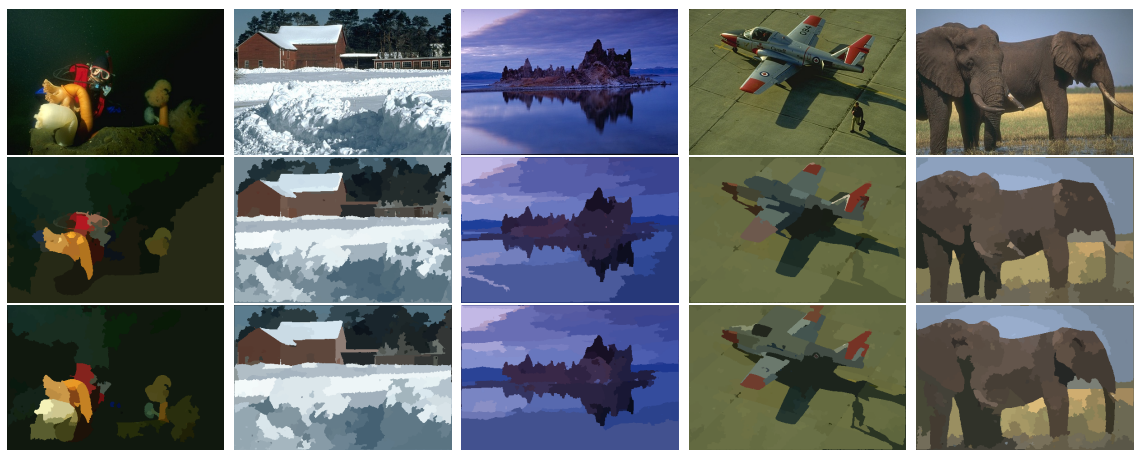

Fig. 1. Segmented images with the combination using Di-Zenzo colour gradient and the texture variance gradient (from top to bottom): initial images, segmented images with the fixed combination, segmented images with the proposed approach)

parts of algae (figure 1 1 1) obtained with the proposed approach is better than the segmentation obtained with the fixed combination. One can note the same remark for the man and the aircraft (figure 14), and the proboscis of the elephant (figure 1.5).

\section{Conclusion}

In this paper, we presented a novel segmentation method combining colour and texture informations. The proposed method is based on an eigenvalues analysis and principal components analysis of a 3D points cloud formed by colour and texture attributes. The contribution of this technique is the definition of an adaptive combination of colour and texture gradients. The proposed combination method provides good segmentation results by watershed algorithm. This technique is local since we assign to each pixel the maximum of information provided by combination of the colour and the texture. Furthermore, the proposed combination is non parametric since it does not require any parameter. In future work, we will expand the number of colour and texture gradients and will show the influence of colour spaces. 
Acknowledgements. This research work is funded in the framework of the ViLoc project and supported by the Regional Council of Franche-Comté (France).

\section{References}

1. Martin, D., Fowlkes, C., Tal, D., Malik, J.: A database of human segmented natural images and its application to evaluating segmentation algorithms and measuring ecological statistics. In: Proc. 8th Int'l Conf. Computer Vision (2001)

2. Lo, E.H.S., Pickering, M.R., Frater, M.R., Arnold, J.F.: Image segmentation using invariant texture features from the double dyadic dual-tree complex wavelet transform. In: IEEE International Conference on Acoustics, Speech and Signal Processing, vol. 1, pp. I-609-I-612 (2007)

3. Paulhac, L., Makris, P., Gregoire, J.-M., Ramel, J.-Y.: Descripteurs de textures pour la segmentation dimages echographiques $3 \mathrm{~d}$. Congrès des Jeunes Chercheurs en Vision par Ordinateur, ORASIS 2009 (2009)

4. Liu, B., Xian: A texture feature fusion-based segmentation method of sar images. In: Second International Conference on Intelligent Human-Machine Systems and Cybernetics (2010)

5. Meyer, F.: Color image segmentation. In: International Conference on Image Processing and its Applications, pp. 303-306 (1992)

6. Kurugollu, F., Sankur, B., Harmanci, A.: Color image segmentation using histogram multithresholding and fusion. Image Vision Computing 19, 915-928 (2001)

7. Delon, J., Desolneux, A., Lisani, J.L., Petro, A.B.: Color image segmentation using acceptable histogram segmentation. In: Pattern Recognition and Image Analysis, pp. 239-246 (2006)

8. Kiranyaz, S., Birinci, M., Gabbouj, M.: Perceptual color descriptor based on spatial distribution: a top-down approach. Image and Vision Computing 28, 1309-1326 (2010)

9. Dubuisson-Jolly, M.-P., Gupta, A.: Color and texture fusion: application to aerial image segmentation and gis updating. Image and Vision Computer 18, 823-832 (2000)

10. Chen, J., Pappas, N., Mojsilovic, A., Rogowitz, B.E.: Adaptive perceptual colortexture image segmentation. IEEE Transactions on Image Processing 14, 1-13 (2005)

11. Ilea, D.E., Whelan, P.F.: Image segmentation based on the integration of colourtexture descriptors. Pattern Recognition 44, 2479-2501 (2011)

12. Vincent, L., Soille, P.: Watersheds in digital spaces: an efficient algorithm based on immersions simulations. IEEE Transactions On Pattern Analysis and Machine Intelligence (PAMI) 13(16), 583-598 (1991)

13. Shafarenko, L., Petrou, M., Kittler, J.: Automatic watershed segmentation of randomly textured color images. IEEE Transactions On Image Processing 6(11), 15301543 (1997)

14. Palm, C., Keysers, D., Lehmann, T., Spitzer, K.: Gabor filtering of complex hue saturation images for color texture classification. In: Proceedings of the 5th Joint Conference on Information Science, pp. 45-49 (2000)

15. Palm, C., Lehmann, T.: Classification of color textures by gabor filtering. Machine Graphics \& Vision International Journal 11, 195-219 (2002)

16. Haralick, R., Shanmugan, K., Dinstein, I.: Textural features for image classification. IEEE Transactions on Systems, Man and Cybernetics 3(6), 610-621 (1973) 
17. Majdoulayne, H.: Extraction de charactéristiques de texture pour la classification dimages satellites. Ph.D. dissertation, Université de Toulouse (2009)

18. Soille, P.: Morphological image analysis, 2nd edn. Springer, Heidelberg (1999)

19. Zenzo, S.D.: A note on the gradient of multi-image. Computer Vision, Graphics, and Image Processing 33, 116-125 (1986)

20. Angulo, J.: Morphological texture gradients. Application to colour+texture watershed segmentation. In: Proc. of the 8th International Symposium on Mathematical Morphology, pp. 399-410 (October 2007)

21. Arbelaez, P., Maire, M., Fowlkes, C., Malik, J.: Contour detection and hierarchical image segmentation. University of California at Berkley, Tech. Rep., February 16 (2010), http://www . eecs . berkley.edu/Pubs/TechRpts/2010/EECS-2010-17.html

22. Martin, D.R., Fowlkes, C.C., Malik, J.: Learning to detect natural image boundaries using local broghtness, color and texture cues. IEEE Transactions On Pattern Analysis and Machine Intelligence 26, 530-549 (2004)

23. Duby, C., Robin, S.: Analyse en composantes principales. Institut National Agronomique Paris - Grignon, Tech. Rep., département O.M.I.P, Institut National Agronomique Paris - Grignon (2006)

24. Gergaud, J.: Unité fondamentale: Algèbre linéaire: une application l'analyse en composantes principales. INP ENSAT, Tech. Rep. (2006)

25. Zeng, W., Zhang, Y.: A novel improvement to pca for image classification. In: International Conference on Computer Science and Service System (CSSS), pp. 1964-1967 (2011)

26. Du, Q., Zhu, W., Yang, H., Fowler, J.E.: Segmented principal component analysis for parallel compression of hyperspectral imagery. IEEE Geoscience and Remote Sensing Letters 6, 713-717 (2009)

27. Ho, P.-M., Wong, T.-T., Leung, C.-S.: Compressing the illumination-adjustable images with principal componenet analysis. IEEE Transaction on Circuits and Systems for Video Technology 15, 355-364 (2005)

28. Ilin, A., Raiko, T.: Practical approaches to principal componenet analysis in the presence of missing values. Journal of Machine Learning Research 11 (2010)

29. Meila, M.: Comparing clusterings: An axiomatic view. In: ICML (2005)

30. Unnikrisshnan, R., Pantofru, C., Hebert, M.: Toward objective evaluation of image segmentation algorithms. PAMI (2007) 